ITP-SB-00-80

\title{
T-duality of the Green-Schwarz superstring
}

\author{
Bogdan Kulik ${ }^{1} \quad$ Radu Roiban² \\ C.N. Yang Institute for Theoretical Physics \\ SUNY at Stony Brook \\ NY11794-3840, USA
}

\begin{abstract}
We study T-duality in the Green-Schwarz formalism to all orders in superspace coordinates. We find two analogs of Buscher rules for the supervielbein and clarify their meaning from the superstring point of view. The transformation rules for the dilaton, spin $1 / 2$ fermions and Ramond-Ramond superfields are also derived.
\end{abstract}

\footnotetext{
${ }^{1}$ E-mail: bkulik@insti.physics.sunysb.edu

${ }^{2}$ E-mail: roiban@insti.physics.sunysb.edu
} 


\section{Introduction}

Since its discovery, T-duality has been studied from various points of view. Together with the other duality symmetries, it has been the source of many recent developments in string theory. Being based on manipulations that do not change the corresponding conformal field theory, T-duality is probably the most securely founded. First identified in the sigma-model [1] and at the level of perturbative string spectra [2], it is believed to be an exact symmetry of string theory. Moreover, it has been shown [3], [4] that the low energy effective actions of type II string theories posses this symmetry.

The action of T-duality on the NS-NS sector of string theory can be constructed rather easily in the NSR formalism. However, the proper treatment of the RR potentials in this formalism is still unknown. Therefore, one has to find alternative approaches to study their transformation under T-duality. One possible approach is to study the vertex operators associated with the RR fields and from there to infer their transformations. This has been considered in [5] and [6]. In [5] the authors have also considered the 9dimensional spectrum of BPS states realized as wrapped $M 2$ branes. The analysis is based on $N=2, D=9$ supersymmetry algebra. The results give further support for the existence of a M-theory/IIB duality [7] that can be understood in terms of a fundamental supermembrane.

Another approach, considered in [3], [G], consists in studying the low energy effective actions of type II string theories, IIA/B supergravities, and constructing the explicit map between their field content. Since the supergravity theories contain informations about world sheet quantum corrections, it necessarily takes into account the quantum corrected T-duality, that is the dilaton shift. This approach considers all orders in superspace coordinates. A hybrid of the previous two approaches was considered in [8], [9]. There, the author considers the action of T-duality on space-time spinors (gravitini, dilatini and supersymmetry parameters) and from there infers the transformation rules for RR fields. Yet another approach considers the Green-Schwarz superstring up to some given order in anticommuting superspace coordinates [10]. Here the authors considered terms up to second order in $\theta$ in the IIA superstring theory. Such terms involve the R-R field couplings. Their transformation under T-duality has been constructed and the IIB action up to $\mathcal{O}\left(\theta^{2}\right)$ has been written down. T-duality in massive type II theories has also been analyzed. Duality to all orders in the odd superspace coordinates for the heterotic string was considered in [11].

In this note we study T-duality in the Green-Schwarz formalism, to all orders in superspace coordinates. In the following section we consider the Green-Schwarz superstring in flat space and construct in detail the dual theory. We study both the supersymmetry transformations as well as the Siegel symmetry and find the map between the corresponding parameters in the two theories. This will prove to be a useful guide in studying the Green-Schwarz superstring in a curved background, which we consider in the next section.

Using the Siegel symmetry and super-covariance requirements we construct the action of 
T-duality on the supervielbein components carrying flat bosonic indices. By additionally requiring that T-duality is an involution, we obtain the Buscher rules for the full supervielbein up to an arbitrary function. By this time the Siegel symmetry transformations will have the right form to imply the conventional constraints as well as the other lower dimensional constraints of the corresponding supergravity theory. On the other hand, these constraints can be explicitly computed using the dual fields. Requiring that they are indeed satisfied fixes the arbitrary function mentioned above. These constraints together with the Bianchi identities imply [14] all the other supergravity constraints. By explicitly computing them in terms of the dual fields we find the T-duality action on the dilaton and axion and their superparteners as well as the T-duality action on the RR field strengths. This will be done in section 4 .

Our result will be that the supervielbein and super two-form the fields of the two type II theories are related by T-duality in the following way: (the other transformations are listed in section 4)

-1 if the duality transformation changes the chirality of the second space-time gravitino then

$$
\begin{array}{rlrl}
\tilde{E}_{(+) 9}{ }^{a} & =\frac{1}{G_{99}} E_{9}{ }^{a} & ; & \tilde{E}_{(+) \hat{M}}{ }^{a}=E_{\hat{M}}{ }^{a}-\frac{G_{\hat{M} 9}}{G_{99}} E_{9}{ }^{a}+\frac{\mathcal{B}_{\hat{M} 9}}{G_{99}} E_{9}{ }^{a} \\
\tilde{E}_{(+) 9}{ }^{1 \alpha} & =-\frac{E_{9}{ }^{1 \beta}}{G_{99}}\left(\Gamma_{-}\right)_{\beta}{ }^{\alpha} ; & \tilde{E}_{(+) 9}{ }^{2 \alpha}=\frac{E_{9}{ }^{2 \beta}}{G_{99}}\left(E_{9}{ }^{a} \Gamma^{a} \Gamma_{-}\right)_{\beta}{ }^{\alpha} \\
\tilde{E}_{(+) \hat{M}}{ }^{1 \alpha} & =\left(E_{\hat{M}}{ }^{1 \beta}-\frac{G_{\hat{M} 9}}{G_{99}} E_{9}{ }^{1 \beta}-\frac{\mathcal{B}_{\hat{M} 9}}{G_{99}} E_{9}{ }^{1 \beta}\right)\left(\Gamma_{-}\right)_{\beta}{ }^{\alpha} \\
\tilde{E}_{(+) \hat{M}}{ }^{2 \alpha} & =\left(E_{\hat{M}}{ }^{2 \beta}-\frac{G_{\hat{M} 9}}{G_{99}} E_{9}{ }^{2 \beta}+\frac{\mathcal{B}_{\hat{M} 9}}{G_{99}} E_{9}{ }^{2 \beta}\right)\left(E_{9}{ }^{a} \Gamma^{a} \Gamma_{-}\right)_{\beta}{ }^{\alpha} \\
\tilde{\mathcal{B}}_{(+) 9 \hat{M}} & =-\tilde{\mathcal{B}}_{(+) \hat{M} 9}=-\frac{G_{9 \hat{M}}}{G_{99}} ; \quad ; \quad \tilde{\mathcal{B}}_{(+) \hat{M} \hat{N}}=\mathcal{B}_{\hat{M} \hat{N}}+\frac{1}{G_{99}}\left[\mathcal{B}_{9 \hat{M}} G_{9 \hat{N}}-G_{9 \hat{M}} \mathcal{B}_{9 \hat{N}}\right)(1.1)
\end{array}
$$

-2 if the duality transformation changes the chirality of the first space-time gravitino then

$$
\begin{aligned}
& \tilde{E}_{(-) 9}{ }^{a}=\frac{1}{G_{99}} E_{9}{ }^{a} \\
& \tilde{E}_{(-) \hat{M}}{ }^{a}=E_{\hat{M}}{ }^{a}-\frac{G_{\hat{M} 9}}{G_{99}} E_{9}{ }^{a}-\frac{\mathcal{B}_{\hat{M} 9}}{G_{99}} E_{9}{ }^{a} \\
& \tilde{E}_{(-) 9}^{1 \alpha}=\frac{E_{9}^{1 \beta}}{G_{99}}\left(E_{9}{ }^{a} \Gamma^{a} \Gamma_{+}\right)_{\beta}^{\alpha} ; \quad \tilde{E}_{(-) 9}^{2 \alpha}=-\frac{E_{9}^{2 \beta}}{G_{99}}\left(\Gamma_{+}\right)_{\beta}{ }^{\alpha} \\
& \tilde{E}_{(-) \hat{M}}^{1 \alpha}=\left(E_{\hat{M}}^{1 \beta}-\frac{G_{\hat{M} 9}}{G_{99}} E_{9}^{1 \beta}-\frac{\mathcal{B}_{\hat{M} 9}}{G_{99}} E_{9}{ }^{1 \beta}\right)\left(E_{9}{ }^{a} \Gamma^{a} \Gamma_{+}\right)_{\beta}{ }^{\alpha} \\
& \tilde{E}_{(-) \hat{M}}{ }^{2 \alpha}=\left(E_{\hat{M}}^{2 \beta}-\frac{G_{\hat{M} 9}}{G_{99}} E_{9}{ }^{2 \beta}+\frac{\mathcal{B}_{\hat{M} 9}}{G_{99}} E_{9}{ }^{2 \beta}\right)\left(\Gamma_{+}\right)^{\alpha} \\
& \tilde{\mathcal{B}}_{(-) 9 \hat{M}}=-\tilde{\mathcal{B}}_{(-) \hat{M} 9}=\frac{G_{9 \hat{M}}}{G_{99}} \quad ; \quad \tilde{\mathcal{B}}_{(-) \hat{M} \hat{N}}=\mathcal{B}_{\hat{M} \hat{N}}+\frac{1}{G_{99}}\left[\mathcal{B}_{9 \hat{M}} G_{9 \hat{N}}-G_{9 \hat{M}} \mathcal{B}_{9 \hat{N}}\right](
\end{aligned}
$$

In the above formula the indices $1 \alpha$ and $2 \alpha$ are the two possible types of spinor indices according to whether the theory is type IIA/B, and $G_{M N}$ is defined as $E_{M}{ }^{a} E_{N}{ }^{b} \eta_{a b}$. These are the Buscher rules at the level of supervielbein super-two forms are related by the 
usual rules. In all our manipulation we absorb the dilaton superfield by rescaling the super-vielbeine. In this setup the dilaton transformation is already encoded in (1.1-1.2), as noted also in [10]. We will rederive it in section 4 from the matching of constraints. We will end with some comments and conclusions.

\section{T-duality in flat space}

Let us consider first the Green-Schwarz superstring in flat space, with the action given by:

$$
\begin{aligned}
S= & -\frac{1}{2 \pi} \int d \tau d \sigma \sqrt{-h} \Pi_{i}{ }^{\mu} \Pi_{j}{ }^{\nu} h^{i j} \eta_{\mu \nu}- \\
& -\frac{1}{\pi} \int d \tau d \sigma \epsilon^{i j}\left[\partial_{i} X^{\mu}\left(\bar{\theta}^{1} \Gamma^{\nu} \partial_{j} \theta^{1}-\bar{\theta}^{2} \Gamma^{\nu} \partial_{j} \theta^{2}\right)+\bar{\theta}^{1} \Gamma^{\nu} \partial_{i} \theta^{1} \bar{\theta}^{2} \Gamma^{\nu} \partial_{j} \theta^{2}\right] \eta_{\mu \nu}
\end{aligned}
$$

with the supervielbein pullback

$$
\Pi_{i}{ }^{\mu}=\partial_{i} X^{\mu}-\bar{\theta}^{1} \Gamma^{\mu} \partial_{i} \theta^{1}-\bar{\theta}^{2} \Gamma^{\mu} \partial_{i} \theta^{2}
$$

We proceed in the standard way ([16], [1]), set $A_{i}=\partial_{i} X^{9}$ and introduce a Lagrange multiplier $\tilde{X}$ to enforce the constraint that $A_{i}$ is flat. We split the action $S$ into $S_{9}\left(A_{i}\right)$ and $\hat{S}$ independent of $A_{i}$ and add

$$
S_{a u x}=\frac{1}{\pi} \int d \tau d \sigma \tilde{X} \epsilon^{i j} \partial_{i} A_{j}
$$

By requiring supersymmetry invariance of the modified action and keeping in mind that $\delta A_{i}=\partial_{i} \delta X^{9}$ one gets the supersymmetry variation of the Lagrange multiplier $\tilde{X}$. Obvi-

ously, $\delta_{\text {susy }} \hat{S}=0$. Thus, the variation of the Lagrange multiplier comes from $\delta_{\text {susy }} S_{9}$ and it reads:

$$
\delta \tilde{X}=\bar{\epsilon}^{1} \Gamma^{\nu} \theta^{1}-\bar{\epsilon}^{2} \Gamma^{\nu} \theta^{2}
$$

To construct the dual action we integrate out $A_{i}$; at the classical level we use its field equation. Since we are in flat space, there are no quantum corrections at one loop level. The resulting action is:

$$
\begin{aligned}
\tilde{S}= & -\frac{1}{2 \pi} \int d \tau d \sigma \sqrt{-h} \tilde{\Pi}_{i}{ }^{\mu} \tilde{\Pi}_{j}{ }^{\nu} h^{i j} \eta_{\mu \nu}- \\
& -\frac{1}{\pi} \int d \tau d \sigma \epsilon^{i j}\left[\partial_{i} \check{X}^{\mu}\left(\bar{\theta}^{1} \Gamma^{\nu} \partial_{j} \theta^{1}-(-)^{\delta_{\nu 9}} \bar{\theta}^{2} \Gamma^{\nu} \partial_{j} \theta^{2}\right)+\right. \\
& \left.+(-)^{\delta_{\nu 9}} \bar{\theta}^{1} \Gamma^{\nu} \partial_{i} \theta^{1} \bar{\theta}^{2} \Gamma^{\nu} \partial_{j} \theta^{2}\right] \eta_{\mu \nu} \\
& \tilde{\Pi}_{i}{ }^{\nu}=(\eta)^{\delta_{\nu 9}}\left(\partial_{i} \check{X}^{\nu}-\bar{\theta}^{1} \Gamma^{\nu} \partial_{i} \theta^{1}-(-)^{\delta_{\nu 9}} \bar{\theta}^{2} \Gamma^{\nu} \partial_{i} \theta^{2}\right)
\end{aligned}
$$

where $\check{X}^{\nu}=\left(X^{\hat{\nu}}, \tilde{X}\right)$ and we introduce $\eta= \pm 1$. 
Requiring covariance for $\tilde{\Pi}_{i}{ }^{\nu}$ expressed in terms of the dual variables, we are led to the following redefinitions:

$$
\eta=+1:\left\{\begin{array}{rl}
\tilde{X}^{\mu} & =\left(X^{\hat{\mu}}, \tilde{X}\right) \\
\tilde{\theta}^{1} & =\theta^{1} \\
\tilde{\theta}^{2} & =\Gamma^{9} \theta^{2}
\end{array} \quad \eta=-1: \quad\left\{\begin{aligned}
\tilde{X}^{\mu} & =\left(X^{\hat{\mu}},-\tilde{X}\right) \\
\tilde{\theta}^{1} & =\Gamma^{9} \theta^{1} \\
\tilde{\theta}^{2} & =\theta^{2}
\end{aligned}\right.\right.
$$

In terms of these variables $\tilde{\Pi}_{i}{ }^{\mu}$ is covariant and has the same form as $\Pi_{i}{ }^{\mu}$. Therefore, the dual action has the same form as the original one. These transformations flipped the chirality of one of the spinor variables, thus interchanging the two type II theories in flat space.

Let us now consider the Siegel symmetry transformation of the initial action and its dual. Following the obvious statement that the variation of a Lagrangian is a sum of variations of fields times their equations of motion, we first write down these equations. Hatted indices run from 0 to 8 .

$$
\begin{aligned}
& 0=\pi \frac{\delta L}{\delta X^{\hat{\mu}}}=\partial_{i}\left(\sqrt{-h} h^{i j} \Pi_{j}^{\hat{\mu}}\right)+\epsilon^{i j}\left(\partial_{i} \bar{\theta}^{1} \Gamma^{\hat{\mu}} \partial_{j} \theta^{1}-\partial_{i} \bar{\theta}^{2} \Gamma^{\hat{\mu}} \partial_{j} \theta^{2}\right) \\
& 0=\pi \frac{\delta L}{\delta A_{i}}=\epsilon^{i j} \partial_{j} \tilde{X}-\sqrt{-h} h^{i j} \Pi_{j}{ }^{9}-\epsilon^{i j}\left(\partial_{i} \bar{\theta}^{1} \Gamma^{9} \partial_{j} \theta^{1}-\partial_{i} \bar{\theta}^{2} \Gamma^{9} \partial_{j} \theta^{2}\right) \\
& 0=-2 \pi \frac{\delta L}{\delta \bar{\theta}^{1}}=-2 \pi \Gamma^{\hat{\mu}} \theta^{1}\left(\frac{\delta L}{\delta X^{\hat{\mu}}}\right)+2 \pi \Gamma^{9} \theta^{1} \partial_{i}\left(\frac{\delta L}{\delta A_{i}}\right)-2 \epsilon^{i j} \partial_{i} A_{j} \Gamma^{9} \theta^{1}- \\
& -8 \sqrt{-h} P_{-}{ }^{i j}\left(\Pi_{i}{ }^{\hat{\mu}} \Gamma_{\hat{\mu}} \partial_{j} \theta^{1}+\Pi_{i}{ }^{9} \Gamma_{9} \partial_{j} \theta^{1}\right), \\
& 0=-2 \pi \frac{\delta L}{\delta \bar{\theta}^{2}}=-2 \pi \Gamma^{\hat{\mu}} \theta^{2}\left(\frac{\delta L}{\delta X^{\hat{\mu}}}\right)+2 \pi \Gamma^{9} \theta^{2} \partial_{i}\left(\frac{\delta L}{\delta A_{i}}\right)-2 \epsilon^{i j} \partial_{i} A_{i} \Gamma^{9} \theta^{2}- \\
& -8 \sqrt{-h} P_{+}{ }^{i j}\left(\Pi_{i}{ }^{\hat{\mu}} \Gamma_{\hat{\mu}} \partial_{j} \theta^{2}+\Pi_{i}{ }^{9} \Gamma_{9} \partial_{j} \theta^{2}\right) \text {. }
\end{aligned}
$$

Using the Siegel transformations of $X^{\hat{\mu}}$

$$
\delta_{\kappa} X^{\hat{\mu}}=-\delta_{\kappa} \bar{\theta}^{I} \Gamma^{\hat{\mu}} \theta^{I}
$$

as well as the variation of the vector field $A_{i}$

$$
\delta_{\kappa} A_{i}=-\partial_{i}\left(\delta_{\kappa} \bar{\theta}^{I} \Gamma^{9} \theta^{I}\right)
$$

we get the variation of the Lagrange multiplier:

$$
\delta_{\kappa} \tilde{X}=\eta \delta_{\kappa} \tilde{X}^{9}=-\delta_{\kappa} \bar{\theta}^{1} \Gamma^{9} \theta^{1}+\delta_{\kappa} \bar{\theta}^{2} \Gamma^{9} \theta^{2}
$$

We also have to express the variation of the spinor coordinates in terms of the dual variables. Originally we had:

$$
\delta_{\kappa} \theta^{I}=\Pi_{i}{ }^{\hat{\mu}} \Gamma_{\hat{\mu}} \kappa^{i I}+\Pi_{i}^{9} \Gamma_{9} \kappa^{i I}
$$


Using $A_{i}$ field equation one can express $\Pi_{i}{ }^{9}$ in terms of the dual variables as follows:

$$
\Pi_{i}{ }^{9}=h_{i j} \frac{\epsilon^{j l}}{\sqrt{-h}}\left[\partial_{l} \tilde{X}-\left(\bar{\theta}^{1} \Gamma^{9} \partial_{l} \theta^{1}-\bar{\theta}^{1} \Gamma^{9} \partial_{l} \theta^{1}\right)\right]=\eta h_{i j} \frac{\epsilon^{j l}}{\sqrt{-h}} \tilde{\Pi}_{l}{ }^{9} .
$$

Using the constraints on the parameters $\kappa^{1 i}$ and $\kappa^{2 i}$ we can write

$$
\Pi_{i}^{9} \Gamma_{9} \kappa^{i 1}=-\eta \tilde{\Pi}_{j}^{9} \Gamma_{9} \frac{\epsilon^{j l}}{\sqrt{-h}} h_{l m} \kappa^{m 1}=\eta \tilde{\Pi}_{j}^{9} \Gamma_{9} \kappa^{j 1}
$$

and

$$
\Pi_{i}^{9} \Gamma_{9} \kappa^{i 2}=-\eta \tilde{\Pi}_{j}^{9} \Gamma_{9} \frac{\epsilon^{j l}}{\sqrt{-h}} h_{l m} \kappa^{m 2}=-\eta \tilde{\Pi}_{j}^{9} \Gamma_{9} \kappa^{j 2} .
$$

Combining (2.14) and (2.15) together with (2.7), (2.9) and (2.11), one arrives at the conclusion that the parameter of the Siegel transformation transforms under T-duality as follows:

$$
\kappa^{i 2} \longrightarrow \tilde{\kappa}^{i 2}=-\Gamma^{9} \kappa^{i 2}, \quad \kappa^{i 1} \longrightarrow \tilde{\kappa}^{i 1}=-\Gamma^{9} \kappa^{i 1}
$$

for $\eta=+1$ and $\eta=-1$, respectively. Obviously, all its properties are the same as in the initial theory except its chirality which was flipped. The redefinitions found above will serve as a guide in considering type II superstring in arbitrary supergravity background.

\section{$3 \quad$ Arbitrary supergravity background}

We now turn to the type II theories in arbitrary supergravity background. Since the explicit form of supervielbein and NS-NS super-two-form are not known to all orders in the odd superspace coordinate, one has to use the superspace constraints to show that under T-duality the type II theories are mapped into each other. The same procedure allows us to construct the Buscher rules at the level of supervielbein. These rules, together with those for the NS-NS super-two-form, implicitely contain the T-duality transformations for all fields in the theory.

It is well known $[10,11]$ that the Green-Schwarz superstring is invariant under the Siegel transformations if the supervielbein and the super-two-form satisfy the supergravity constraints. At the same time however, invariance under Siegel transformations only requires that certain linear combinations of supergravity constraints hold. Therefore, our strategy will be the following: first we study the Siegel transformations in the dual theory; Combining them with covariance requirements we will construct four possible vielbein solutions of the Buscher rules for the "super-metric"; Requiring further that Tduality is an involution we will discard two of the possible vielbein with flat bosonic index and we will show that the vielbein with flat spinor indices can be redefined such that the Siegel transformations have the standard form. This determines all the components of the supervielbein up to an arbitrary function. This function will be determined by explicitly computing the constraints required by the Siegel transformations in terms of 
the dual fields and requiring that they are satisfied. The other constraints follow from Bianchi identities as shown in [14]. We will use them to derive the transformation of fields that do not appear explicitly in the $\sigma$ model.

Consider the type IIA action in a general supergravity background, which can be obtained by dimensional reduction of 11-dimensional supermembrane action [15], and assume that all fields are independent on the coordinate $X^{9}$. Replacing $\partial_{i} X^{9}$ by the vector field $A_{i}$ and constraining it to be flat we get the action:

$$
\begin{aligned}
S & =\int d^{2} \xi\left(\frac{1}{2} \Phi \sqrt{-g} g^{i j} E_{i}{ }^{a} E_{j}{ }^{b} \eta_{a b}+\epsilon^{i j} \partial_{i} Z^{M} \partial_{j} Z^{N} \mathcal{B}_{N M}+\epsilon^{i j} \tilde{X} \partial_{i} A_{j}\right) \\
\mathcal{B}_{M N} & =(-)^{M(N+A)} E_{N}{ }^{A} E_{M}{ }^{B} \mathcal{B}_{B A} \quad \text { and } \quad E_{i}{ }^{a} \equiv A_{i} E_{9}{ }^{a}+\partial_{i} Z^{\hat{M}} E_{\hat{M}}{ }^{a}
\end{aligned}
$$

The dilaton superfield $\Phi$ can be removed by a suitable rescaling of the supervielbein, so we will set it equal to 1 in the following.

Defining:

$$
G_{99}=E_{9}{ }^{a} E_{9}{ }^{b} \eta_{a b}, \quad G_{9 \hat{M}}=E_{9}{ }^{a} E_{\hat{M}}^{b} \eta_{a b}, \quad G_{\hat{M} \hat{N}}=E_{\hat{M}}^{a} E_{\hat{N}}^{b} \eta_{a b}
$$

integrating out $A_{i}$ using its field equation

$$
E_{i}{ }^{a}=\partial_{i} Z^{\hat{M}}\left(E_{\hat{M}}{ }^{a}-\frac{G_{\hat{M} 9}}{G_{99}} E_{9}{ }^{a}\right)-\frac{g_{i j} j^{j k}}{\sqrt{-g} G_{99}}\left(\partial_{k} \tilde{X}+\partial_{k} Z^{\hat{M}} \mathcal{B}_{\hat{M} 9}\right) E_{9}{ }^{a}
$$

and allowing for the identifications $\tilde{X}^{9}=\eta \tilde{X}$ with $\eta= \pm 1$, we get:

-dual "supermetric":

$$
\begin{aligned}
\tilde{G}_{99} & =\frac{1}{G_{99}} \\
\tilde{G}_{9 \hat{M}} & =-\frac{\eta}{G_{99}} \mathcal{B}_{9 \hat{M}}=\tilde{G}_{\hat{M} 9} \\
\tilde{G}_{\hat{M} \hat{N}} & =G_{\hat{M} \hat{N}}-\frac{1}{G_{99}}\left[G_{9 \hat{M}} G_{9 \hat{N}}-\mathcal{B}_{9 \hat{M}} \mathcal{B}_{9 \hat{N}}\right]
\end{aligned}
$$

-dual super 2-form:

$$
\begin{aligned}
\tilde{\mathcal{B}}_{9 \hat{M}} & =-\frac{\eta}{G_{99}} G_{9 \hat{M}}=-\tilde{\mathcal{B}}_{\hat{M} 9} \\
\tilde{\mathcal{B}}_{\hat{M} \hat{N}} & =\mathcal{B}_{\hat{M} \hat{N}}+\frac{1}{G_{99}}\left[\mathcal{B}_{9 \hat{M}} G_{9 \hat{N}}-G_{9 \hat{M}} \mathcal{B}_{9 \hat{N}}\right] .
\end{aligned}
$$

Following the same steps as in flat space and using the Siegel transformations of the IIA theory [12] we deduce the Siegel transformation of the $\tilde{X}^{9}$ coordinate:

$$
\delta_{\kappa} \tilde{X}^{9}=-\eta \delta_{\kappa} Z^{\hat{M}} \mathcal{B}_{\hat{M} 9}
$$


Let us now check whether the dualized variables obey the type IIB Siegel transformation rules. First we notice that using the Siegel symmetry of type IIA theory, $\delta_{\kappa} E^{a} \equiv \delta_{\kappa} Z^{M} E_{M}{ }^{a}=0$, one can write:

$$
\delta_{\kappa} Z^{M} G_{M N}=0 \Longrightarrow \delta_{\kappa} Z^{\hat{M}} G_{\hat{M} N}=-\delta_{\kappa} X^{9} G_{9 N}
$$

which together with 3.5 and 3.6 leads to

$$
\begin{aligned}
\delta_{\kappa} \tilde{Z}^{N} \tilde{E}_{N}{ }^{a} \tilde{E}_{\hat{M}}^{b} \eta_{a b} & =\delta_{\kappa} \tilde{X}^{9} \tilde{G}_{9 \hat{M}}+\delta_{\kappa} Z^{\hat{N}} \tilde{G}_{\hat{N} \hat{M}}= \\
& =-\eta \delta_{\kappa} Z^{\hat{N}} \mathcal{B}_{\hat{N} 9} \frac{\eta}{G_{99}} \mathcal{B}_{\hat{M} 9}+\delta_{\kappa} Z^{\hat{N}}\left[G_{\hat{N} \hat{M}}-\frac{1}{G_{99}}\left(G_{9 \hat{N}} G_{9 \hat{M}}-\mathcal{B}_{9 \hat{N}} \mathcal{B}_{9 \hat{M}}\right)\right]= \\
& =\delta_{\kappa} Z^{\hat{N}}\left[G_{\hat{N} \hat{M}}-\frac{1}{G_{99}} G_{9 \hat{N}} G_{9 \hat{M}}\right] .
\end{aligned}
$$

The contraction with $E_{9}{ }^{a}$ must be considered separately and we obtain

$$
\delta_{\kappa} \tilde{Z}^{N} \tilde{E}_{N}{ }^{a} \tilde{E}_{9}{ }^{b} \eta_{a b}=-\eta \delta_{\kappa} Z^{\hat{M}} \mathcal{B}_{\hat{M} 9} \tilde{G}_{99}+\delta_{\kappa} Z^{\hat{M}} \tilde{G}_{\hat{M} 9}=0 .
$$

Multiplying by $\tilde{E}_{a}{ }^{M}$, we find

$$
\delta_{\kappa} \tilde{Z}^{N} \tilde{E}_{N}^{a}=0
$$

Notice that in deriving this result we did not need to know the action of T-duality at the level of supervielbein. This is, however, not a generic feature as we will see by considering the spinor part of Siegel transformations.

Start from the IIA theory and introduce two vielbeine, $E_{M}{ }^{I \alpha}, I=1,2$, subject to chirality constraints $E_{M}{ }^{1 \alpha}\left(\Gamma_{+}\right)_{\alpha}{ }^{\beta}=0$ and $E_{M}{ }^{2 \alpha}\left(\Gamma_{-}\right)_{\alpha}{ }^{\beta}=0$. Here $\alpha$ denotes a 10-dimensional spinor index, $\alpha \equiv(\dot{\alpha}, \alpha)$ and $\Gamma_{ \pm}=\frac{1}{2}\left(1 \pm \Gamma_{11}\right)$. The Siegel symmetry transformations of IIA theory are given by [15]:

$$
\delta_{\kappa} E^{1 \alpha}=E_{i}{ }^{a} P_{+}{ }^{i j} \kappa_{j}{ }^{1 \rho}\left(\Gamma_{+}\right)_{\rho}{ }^{\beta}\left(\Gamma_{a}\right)_{\beta}{ }^{\alpha} \quad, \quad \delta_{\kappa} E^{2 \alpha}=E_{i}{ }^{a} P_{-}{ }^{i j} \kappa_{j}{ }^{2 \rho}\left(\Gamma_{-}\right)_{\rho}{ }^{\beta}\left(\Gamma_{a}\right)_{\beta}{ }^{\alpha}
$$

with the usual $P_{ \pm}{ }^{i j}=\frac{1}{2}\left(g^{i j} \pm \frac{\epsilon^{i j}}{\sqrt{-g}}\right)$ and $\delta_{\kappa} E^{I \alpha} \equiv \delta_{\kappa} Z^{M} E_{M}{ }^{I \alpha}$. Let us also introduce for the dual theory two types of vielbein with flat spinor indices $\left(\tilde{E}^{I \alpha}, I=1,2\right)$. For each of the two vielbeine the Siegel variation is given by:

$\delta_{\kappa} \tilde{E}^{I \alpha} \equiv \delta_{\kappa} \tilde{X}^{9} \tilde{E}_{9}^{I \alpha}+\delta_{\kappa} Z^{\hat{M}} \tilde{E}_{\hat{M}}^{I \alpha}=\delta_{\kappa} Z^{\hat{M}}\left[\tilde{E}_{\hat{M}}^{I \alpha}-\eta \mathcal{B}_{\hat{M} 9} \tilde{E}_{9}^{I \alpha}\right]=\delta_{\kappa} Z^{\hat{M}}\left[\tilde{E}_{\hat{M}}^{I \alpha}-\frac{\tilde{G}_{\hat{M} 9}}{\tilde{G}_{99}} \tilde{E}_{9}^{I \alpha}\right]$,

where we have used (3.6). Let us introduce the notation:

$$
\tilde{P}_{\hat{M}}^{I \alpha}=\tilde{E}_{\hat{M}}^{I \alpha}-\frac{\tilde{G}_{\hat{M} 9}}{\tilde{G}_{99}} \tilde{E}_{9}^{I \alpha} .
$$

From the original theory we have:

$$
\delta_{\kappa} Z^{\hat{M}}=\delta_{\kappa} E^{I \alpha} E_{I \alpha}^{\hat{M}} .
$$


Rewriting the $A_{i}$ field equation (3.3) using the dual variables one gets for $E_{i}{ }^{a}$ :

$$
E_{i}{ }^{a}=\partial_{i} Z^{\hat{M}} E_{\hat{M}}{ }^{a}-\eta\left[g_{i j}\left(P_{+}{ }^{j k}-P_{-}{ }^{j k}\right) \partial_{k} \tilde{Z}^{M} \tilde{G}_{M 9}+\partial_{i} Z^{\hat{M}} \tilde{\mathcal{B}}_{\hat{M} 9}\right] E_{9}{ }^{a}
$$

Thus, recalling that $P_{+}{ }^{i j} P_{-}{ }^{k l} g_{j k}=0$ and $P_{+}{ }^{i j} P_{+}{ }^{k l} g_{j k}=P_{+}{ }^{i l}$ one gets for the variations in the dual theory:

$$
\begin{aligned}
\delta_{\kappa} \tilde{E}^{I \alpha} & =\left\{\partial_{i} Z^{\hat{M}}\left(E_{\hat{M}}{ }^{a}-\eta \tilde{\mathcal{B}}_{\hat{M} 9} E_{9}{ }^{a}\right)+\eta \partial_{i} \tilde{Z}^{M} \tilde{G}_{M 9} E_{9}{ }^{a}\right\} P_{+}^{i j} \kappa_{j}^{1 \rho}\left(\Gamma_{+}\right)_{\rho}{ }^{\beta}\left(\Gamma_{a}\right)_{\beta}{ }^{\sigma} E_{\sigma}{ }^{1 \hat{M}} \tilde{P}_{\hat{M}}{ }^{I \alpha}+ \\
& \left.+\left\{\partial_{i} Z^{\hat{M}}\left(E_{\hat{M}}{ }^{a}-\eta \tilde{\mathcal{B}}_{\hat{M} 9} E_{9}{ }^{a}\right)-\eta \partial_{i} \tilde{Z}^{M} \tilde{G}_{M 9} E_{9}{ }^{a}\right\} P_{-}^{i j} \kappa_{j}^{2 \rho}\left(\Gamma_{-}\right)_{\rho}{ }^{\beta}\left(\Gamma_{a}\right)_{\beta}{ }^{\sigma} E_{\sigma}{ }^{2 \hat{M}} \tilde{P}_{\hat{M}}{ }^{I(3)} .16\right)
\end{aligned}
$$

From the above expressions, on covariance ground, we can make the following identification:

$$
\begin{aligned}
\tilde{E}_{(\sigma, \eta) 9}{ }^{a} & =\sigma \eta \frac{1}{G_{99}} E_{9}{ }^{a}, \\
\tilde{E}_{(\sigma, \eta) \hat{M}}{ }^{a} & =E_{\hat{M}}{ }^{a}-\frac{G_{\hat{M} 9}}{G_{99}} E_{9}{ }^{a}+\sigma|\eta| \frac{\mathcal{B}_{\hat{M} 9}}{G_{99}} E_{9}{ }^{a}
\end{aligned}
$$

where $\sigma= \pm 1$ independently of $\eta$ and the absolute value of $\eta$ in the second equation is for notational convenience. It is easy to check that (3.17) are solutions for (3.4) for all $\sigma$ and $\eta$. These equations represent the action of T-duality on the vielbein components with flat bosonic indices. As one probably expects, using these expressions for the dual vielbein one can immediately get equation (3.10):

$$
\begin{aligned}
\delta_{\kappa} \tilde{E}_{(\sigma, \eta)}{ }^{a} & =\delta_{\kappa} Z^{\hat{M}}\left\{-\sigma \mathcal{B}_{\hat{M} 9} \frac{E_{9}{ }^{a}}{G_{99}}+E_{\hat{M}}{ }^{a}-\frac{G_{\hat{M} 9}}{G_{99}} E_{9}{ }^{a}+\sigma \frac{\mathcal{B}_{\hat{M} 9}}{G_{99}} E_{9}{ }^{a}\right\} \\
& =\delta_{\kappa} Z^{\hat{M}}\left\{E_{\hat{M}}{ }^{a}-\frac{G_{\hat{M} 9}}{G_{99}} E_{9}{ }^{a}\right\}=0
\end{aligned}
$$

upon using (3.7) for the last equal sign. At this point we seem to have four possible choices of vielbein with flat bosonic indices, two for each choice of $\eta$. This is twice as many as in [4], [8], [9], where similar expressions have been considered.

This is as much as we can get by considering only the Siegel transformations and covariance. By requiring that T-duality is an involution we will obtain additional useful informations. It is easy to check that equation (3.17) satisfy the involution requirement for all values of $\sigma$ and $\eta$. However, we will be able to single out a unique vielbein for each choice of $\eta$ by studying the transformations of the $\kappa$ parameters. In [9] it has been argued that both solutions are necessary for the consistency of the theory. As we will see below, the two solutions correspond to IIB theories with opposite chiralities.

The first step is to notice that $(3.16)$ can be cast in a more useful form using the following identity:

$$
\partial_{\kappa} \tilde{Z}^{M} \tilde{E}_{(\sigma, \eta) M}{ }^{a} \Gamma^{a}=-\partial_{\kappa} \tilde{Z}^{M} \tilde{E}_{(-\sigma, \eta) M}{ }^{a} \ddot{H}_{9} \Gamma^{a} \ddot{H}_{9} .
$$

Here we have introduced the notation

$$
\left(E_{9}\right)_{\alpha}{ }^{\beta} \equiv \frac{E_{9}{ }^{a}}{\sqrt{G_{99}}}\left(\Gamma_{a}\right)_{\alpha}{ }^{\beta},
$$


which plays the role of $\Gamma_{9}$ in the flat case above. Thus, with the redefinition

$$
\kappa_{j}^{2 \alpha} \longrightarrow \tilde{\kappa}_{j}^{2 \alpha}=-\kappa_{j}^{2 \beta} E_{9 \beta}{ }^{\alpha}, \quad \kappa_{j}^{1 \alpha} \longrightarrow \tilde{\kappa}_{j}^{1 \alpha}=-\kappa_{j}^{1 \beta} E_{9 \beta}{ }^{\alpha}
$$

for $\sigma=+1$ and $\sigma=-1$ respectively, equation (3.16) can be written as follows:

$$
\begin{aligned}
\delta_{\kappa} \tilde{E}^{I \alpha} & =\partial_{i} \tilde{Z}^{N} \tilde{E}_{(+, \eta)_{N}}{ }^{a}\left\{P_{+}{ }^{i j} \kappa_{j}{ }^{1 \zeta}\left(\Gamma_{a}\right)_{\zeta}{ }^{\beta} E_{1 \beta}{ }^{\hat{M}}+P_{-}{ }^{i j} \tilde{\kappa}_{j}{ }^{2 \zeta}\left(\Gamma_{a}\right)_{\zeta}{ }^{\sigma}\left(E_{9}\right)_{\sigma}{ }^{\beta} E_{2 \beta}{ }^{\hat{M}}\right\} \tilde{P}_{\hat{M}}{ }^{I \alpha} \\
& =\partial_{i} \tilde{Z}^{N} \tilde{E}_{(-, \eta)_{N}}{ }^{a}\left\{P_{+}{ }^{i j} \tilde{\kappa}_{j}{ }^{1 \zeta}\left(\Gamma_{a}\right)_{\zeta}{ }^{\sigma}\left(E_{9}\right)_{\sigma}{ }^{\beta} E_{1 \beta}{ }^{\hat{M}}+P_{-}{ }^{i j} \kappa_{j}{ }^{2 \zeta}\left(\Gamma_{a}\right)_{\zeta}{ }^{\beta} E_{2 \beta}{ }^{\hat{M}}\right\} \tilde{P}_{\hat{M}}{ }^{I \alpha}
\end{aligned}
$$

where we have absorbed the chiral projectors by letting them act on $\kappa$. Notice that the redefinition of $\kappa_{j}^{1(2)}$ is the direct curved space analog of the flat space case. Notice also that equation (3.21) can be written in a more compact form:

$$
\tilde{\kappa}_{(\eta) j}{ }^{I \alpha}=-\kappa_{(\eta) j}{ }^{J \beta} \mathcal{T}_{(\eta)}{ }_{J \beta}{ }^{I \alpha}
$$

with $\mathcal{T}_{(\eta)}{ }_{J \beta}^{I \alpha}$ defined as follows:

$\mathcal{T}_{(+)_{B}}{ }^{A}=\left\{\begin{array}{cll}\delta_{B}{ }^{A} & \text { for } A, B \in\{a, 1 \alpha\} \\ \left(\mathscr{E}_{9}\right)_{\beta}{ }^{\alpha} & \text { for } A, B=2 \alpha\end{array} \quad ; \mathcal{T}_{(-)_{B}}{ }^{A}=\left\{\begin{array}{cl}\delta_{B}{ }^{A} & \text { for } A, B \in\{a, 2 \alpha\} \\ \left(E_{9}\right)_{\beta}{ }^{\alpha} & \text { for } A, B=1 \alpha\end{array}\right.\right.$

A priori there is no principle allowing us to fix $E_{I \beta}{ }^{\hat{M}} \tilde{P}_{\hat{M}}{ }^{J \alpha}$ in terms of the fields of the IIA theory. The strategy for solving this problem will be the following: we parametrize the arbitrariness of the dual vielbein via their projection on the original ones and see how much of it can be accounted for by field redefinitions that do not change the form of the Lagrangian. As stressed previously, we require that the resulting Siegel variations are covariant and that T-duality is an involution up to relabeling of vielbein and $\kappa$ parameters. The remaining pieces could be interpreted as the analog of Buscher rules for the vielbein with flat spinor indices.

Let us consider next the parameters of the Siegel transformations. For $\tilde{E}^{a}=\tilde{E}_{(+, \eta)}{ }^{a}$ we impose that $\tilde{\tilde{\kappa}}^{2}=\kappa^{2}$ and we get $\eta=1$. For the other choice of dual vielbein, $\tilde{E}^{a}=\tilde{E}_{(-, \eta)}{ }^{a}$, we impose that $\tilde{\tilde{\kappa}}^{1}=\kappa^{1}$ and we get $\eta=-1$. These can be summarized in the following equation:

$$
\sigma \eta=1 \Leftrightarrow \sigma=\eta
$$

Thus, as promised, we are left with a unique vielbein with flat bosonic indices for each choice of $\eta$.

Now we analyze the vielbein with flat spinor indices and show that the two values of $\eta$ correspond to the two IIB theories with opposite chiralities, just as in the case of flat space. For notational convenience we will replace the pair $(\sigma, \eta)$ by $\eta$. Since $\tilde{E}_{9}{ }^{a}$ is independent of $\eta$, it will not carry such an index. Let us also associate, in the obvious way, an index $\eta$ to the vielbein with flat spinor indices.

Following the strategy explained above, let us parametrize the dual vielbein via their projection on the original vielbein as follows:

$$
E_{I \alpha}^{\hat{M}} \tilde{P}_{(\eta) \hat{M}}^{J \beta}=\mathcal{M}_{(\eta)}{ }_{I \alpha}{ }^{J \beta}
$$




$$
\begin{aligned}
E_{a}{ }^{\hat{M}} \tilde{P}_{(\eta) \hat{M}}{ }^{J \beta} & =\mathcal{A}_{(\eta)_{a}}{ }^{J \beta}-\eta_{a b} \frac{E_{9}{ }^{b}}{G_{99}} E_{9}^{I \alpha} \mathcal{M}_{(\eta)_{I \alpha}}{ }^{J \beta} \text { with } E_{9}{ }^{a} \mathcal{A}_{(\eta)_{a}}{ }^{I \beta}=0 \\
\tilde{E}_{(\eta)_{9}}{ }^{I \alpha} & =a_{(\eta)}{ }^{I \alpha}(E)
\end{aligned}
$$

where $\mathcal{M}^{I J}, \mathcal{A}$ and $a$ are for the time being arbitrary functions of the IIA fields. The chirality of the original vielbein implies that $\left(\Gamma_{+}\right)_{\alpha}{ }^{\beta} \mathcal{M}_{1 \beta}{ }^{J \gamma}=0$ and $\left(\Gamma_{-}\right)_{\alpha}{ }^{\beta} \mathcal{M}_{2 \beta}{ }^{J \gamma}=0$. Equations (3.26) completely describe $\tilde{E}_{(\eta)_{M}}{ }^{I \alpha}$ as can be seen from the following counting of degrees of freedom: for a given $\eta, \mathcal{M}_{(\eta)}$ with its chirality constraints accounts for $2 \times 2 \times \frac{1}{2} \times 32 \times 32$ independent components while $\mathcal{A}$ and $a$ account for $9 \times 2 \times 32$ and $2 \times 32$, respectively. We have therefore the number of independent components of $\tilde{E}_{(\eta)_{M}}{ }^{I \alpha}$. On the other hand, the duality transformation does not change the number of independent degrees of freedom. The chirality constraints on $\mathcal{M}$ require that its rank is at most 32 which is also the number of degrees of freedom in the original theory. Therefore, we expect that all field redefinitions will involve only non-degenerate matrices.

We can easily invert the equations (3.26) and express $\tilde{P}_{(\eta) \hat{M}}{ }^{I \alpha}$ in terms of IIA fields and the arbitrary objects $\mathcal{M}$ and $\mathcal{A}$. Introducing the notation $P_{\hat{M}}^{I A}=E_{\hat{M}}{ }^{I A}-\frac{G_{\hat{M} 9}}{G_{99}} E_{9}{ }^{I A}$, the solution reads:

$$
\begin{aligned}
\tilde{P}_{(\eta)_{\hat{M}}}^{I \alpha} & =P_{\hat{M}}{ }^{J \beta} \mathcal{M}_{(\eta)_{J \beta}}{ }^{I \alpha}+P_{\hat{M}}^{b} \mathcal{A}_{(\eta)_{b}}{ }^{I \alpha} \\
\tilde{E}_{(\eta)_{9}}{ }^{I \alpha} & =a_{(\eta)}{ }^{I \alpha}(E)
\end{aligned}
$$

where we have also used that $E_{9}{ }^{a} \mathcal{A}_{a}{ }^{I \beta}=0$. This is the most general solution since equations (3.26) form a linear system with as many equations as unknowns. At this point it seems natural to introduce also the object $\tilde{P}_{(\eta) \hat{M}}{ }^{a}=\tilde{E}_{(\eta) \hat{M}}{ }^{a}-\frac{\tilde{G}_{\hat{M} 9}}{\tilde{G}_{99}} \tilde{E}_{(\eta) 9}{ }^{a}$ and notice that it is equal to $P_{\hat{M}}{ }^{a}$ upon using the explicit form (3.17) of vielbein with flat bosonic index. All these can be summarized in the following equation:

$$
\left(\begin{array}{ll}
\tilde{P}_{(\eta) \hat{M}}^{a} & \tilde{P}_{(\eta) \hat{M}}^{I \alpha}
\end{array}\right)=\left(\begin{array}{ll}
P_{\hat{M}}^{b} & P_{\hat{M}}{ }^{J \beta}
\end{array}\right)\left(\begin{array}{cc}
\delta_{b}{ }^{a} & \mathcal{A}_{(\eta)_{b}}{ }^{I \alpha}{ }^{I \alpha} \\
0 & \mathcal{M}_{(\eta)_{J \beta}}
\end{array}\right)
$$

In the following we will enforce the chirality constraints on $\mathcal{M}$ by expressing it as appropriate projectors acting on nondegenerate matrices.

Acting on equation (3.28) from the right with matrices of the type $\left(\begin{array}{ll}\mathbb{1} & C \\ 0 & \mathbb{1}\end{array}\right)$ we notice that the value of $\mathcal{A}$ can be changed arbitrarily. At the same time, such transformations are nondegenerate for any $C$ and leave the Lagrangian invariant. They are, therefore, perfectly valid field redefinitions. Using them we will set $\mathcal{A}=0$ in the following. Notice that in this procedure we did not use the extra constraints coming from Siegel symmetry.

By requiring that equation(3.22) is covariant we see that the matrices $\mathcal{M}$ must be of the form:

$$
\mathcal{M}_{(\eta)}{ }_{I \alpha}{ }^{J \beta}=\mathcal{T}_{(\eta)}{ }_{I \alpha}{ }^{K \gamma} \delta_{K}{ }^{L}\left(\Gamma_{-\eta}\right)_{\gamma}{ }^{\rho} \mathcal{N}_{(\eta)}{ }_{L \rho}{ }^{J \beta}
$$

where $\mathcal{N}$ are arbitrary, covariant, nondegenerate objects, $\mathcal{T}$ was defined in equation (3.24) and $\Gamma_{-\eta}$ is defined to be $\Gamma_{ \pm}$for $\eta=\mp$. We have also explicitely taken into account the 
chirality constraints by inserting the appropriate projectors. We could of course ask ourselves whether there really exists a nontrivial $\mathcal{N}$ constructed out of covariant objects in the theory which also has the right dimensions. As we will see below, there is no need to construct the most general such object since by knowing that it is nondegenerate we can absorb it by redefining $\tilde{E}_{M}^{I \alpha}$. This will completely fix the Siegel symmetry transformations. In the following we will construct the inverse of the matrix $\mathcal{N}$.

Requiring that T-duality is an involution provides additional informations regarding $\mathcal{M}_{(\eta)}$ and $a_{(\eta)}$. Following the same steps as before but this time using (3.22) for the Siegel transformations, we get the following equation:

$$
\mathcal{T}_{(\tilde{\eta})}{ }_{I \alpha}{ }^{K \gamma} \mathcal{T}_{(\eta)}{ }_{K \gamma}{ }^{P \epsilon} \mathcal{M}_{(\eta)}{ }_{P \epsilon}{ }^{L \rho} \tilde{\mathcal{M}}_{(\eta, \tilde{\eta})}{ }_{L \rho}{ }^{J \beta}=\left(\delta_{|\eta-\tilde{\eta}|,|I-J|}\right)_{I}{ }^{J}\left[\left(\Gamma_{-}\right)_{\alpha}{ }^{\beta} \delta_{J}{ }^{1}+\left(\Gamma_{+}\right)_{\alpha}{ }^{\beta} \delta_{J}{ }^{2}\right]
$$

where $\tilde{\mathcal{M}}_{(\eta, \tilde{\eta})}{ }_{I \alpha}{ }^{J \beta}=\tilde{E}_{(\eta))_{I \alpha}}{ }^{\hat{M}}\left(E_{(\tilde{\eta}) \hat{M}}{ }^{J \beta}-\frac{G_{\hat{M} 9}}{G_{99}} E_{(\tilde{\eta})_{9}}{ }^{J \beta}\right), E_{(\tilde{\eta})_{M}}{ }^{I \beta}=E_{M}^{I \beta}$ if $\tilde{\eta}=\eta$ and $E_{(\tilde{\eta})}{ }_{M}^{I \beta}$ for $\tilde{\eta}=-\eta$ will be constructed shortly. One might wonder why we are repeating all the computation and do not formally dualize equation (3.28). The point is that there is additional information, related to covariance requirement, that has to be taken into account.

We claim that it is enough to consider, say, $\tilde{\eta}=\eta$. In order to prove this claim we notice that $\left(\mathcal{T}_{(\eta)}\right)^{2}=\mathbb{1} \otimes \mathbb{1}$ and $\mathcal{T}_{(\eta)} \mathcal{T}_{(-\eta)}=\mathbb{1} \otimes \mathbb{E}_{9}$. We can therefore construct a map between $\tilde{\eta}=-\eta$ and $\tilde{\eta}=\eta$ theories:

$$
\tilde{\mathcal{M}}_{(\eta, \eta)}^{J 1}=\tilde{\mathcal{M}}_{(\eta,-\eta)}^{J 2} E_{9} \quad, \quad \tilde{\mathcal{M}}_{(\eta, \eta)}{ }^{J 2}=\tilde{\mathcal{M}}_{(\eta,-\eta)}{ }^{J 1} \not_{9} .
$$

This immediately translates into a map between the vielbein associated with the $\tilde{\eta}= \pm \eta$ :

$$
E_{(\tilde{\eta}=-\eta)_{M}}^{I \alpha}=E_{(\tilde{\eta}=\eta)_{M}}{ }^{J \beta}\left|\epsilon^{J I}\right|\left(H_{9}\right)_{\beta}{ }^{\alpha}
$$

Of course, this should be combined with a change in sign of the $X^{9}$ coordinate. At the level of Siegel transformations this translates into the statement that $\delta_{\kappa} E_{(\tilde{\eta}=-\eta)}^{I \alpha} \sim\left|\epsilon^{I J}\right| \kappa^{J \alpha}$. Since it seems natural for the index on $E$ to match the index on $\kappa$, we relabel the vielbein to absorb the $\epsilon$ symbol. The relabeled vielbein have the chirality opposite to those obtained by choosing $\tilde{\eta}=\eta$. We therefore conclude that the two IIA theories are mapped into each other by multiplying with $E_{9}$ the vielbein with flat spinor indices and relabeling the result together with performing a parity transformation along the ninth coordinate. The last statement is subject to interpretation since the two IIA theories are actually identical. From this point of view, the map described above is just an automorphism of the IIA theory.

In the following we will concentrate on the choice $\tilde{\eta}=\eta$. Combining equation (3.30) with equation (3.29) we see that, since $\mathcal{N}^{I J}$ is covariant, we need $\tilde{\mathcal{M}}_{(\eta)}{ }^{I J}$ to be proportional to $\tilde{T}_{(\eta)}$. Thus, we are lead to rewrite $\tilde{\mathcal{M}}$ as follows:

$$
\tilde{\mathcal{M}}_{(\eta, \eta)_{I}}{ }^{J}=\tilde{\mathcal{N}}_{(\eta, \eta)_{I}}{ }^{K} \Gamma_{-\eta} \mathcal{T}_{(\eta)_{K}}{ }^{J}
$$

where $\tilde{\mathcal{N}}$ are, for now, arbitrary matrices and we have taken care of chirality constraints. 
Using this expression for $\tilde{\mathcal{M}}$, the equation $(3.30)$ can be written as:

$$
\Gamma_{-\eta} \mathcal{N}_{(\eta)} \tilde{\mathcal{N}}_{(\eta, \eta)} \Gamma_{-\eta}=\mathbb{1} \otimes \Gamma_{-\eta}
$$

This shows that $\tilde{\mathcal{N}}_{(\eta, \eta)_{I}}{ }^{J}$ is the inverse of $\mathcal{N}_{(\eta)_{I}}{ }^{J}$ in the $\Gamma_{-\eta}$ sector and therefore enables us to redefine the vielbein and completely absorb the arbitrariness in $\mathcal{N}^{I J}$. With the vielbein redefinitions

$$
\tilde{E}_{(\eta)_{M}}^{I \alpha} \longrightarrow \tilde{E}_{(\eta)_{M}}^{I \alpha}=\tilde{E}_{(\eta)_{M}}{ }^{J \beta} \tilde{\mathcal{N}}_{(\eta, \eta)_{J \beta}}^{I \alpha}
$$

we therefore get the following dual fields:

$$
\tilde{P}_{(\eta)}^{I \alpha}=P^{J \beta} \mathcal{T}_{(\eta)}{ }_{J \beta}^{I \gamma}\left(\Gamma_{-\eta}\right)_{\gamma}{ }^{\alpha}
$$

or, explicitly,

$$
\tilde{E}_{(\eta) \hat{M}}^{I \alpha}=\left(E_{\hat{M}}^{J \beta}-\frac{G_{\hat{M} 9}}{G_{99}} E_{9}^{J \beta}\right) \mathcal{T}_{(\eta) J \beta}^{I \gamma}\left(\Gamma_{-\eta}\right)_{\gamma}{ }^{\alpha}+\eta B_{\hat{M} 9} \tilde{E}_{(\eta) 9}^{I \alpha}
$$

Thus, we see that all arbitrariness of $\mathcal{M}$ and $\mathcal{A}$ can be removed by suitable field redefinitions. The left over piece in equation (3.35) $\left(\mathcal{T}_{(\eta)_{J \beta}}{ }^{I \gamma}\right)$ is needed to restore the covariance of the dual Siegel transformations. $\tilde{E}_{(\eta) 9}{ }^{I \alpha}$ is left arbitrary by the above analysis, but it will be fixed shortly from other considerations. Its chirality is, however, fixed by equation (3.35). Of course, the chiral projector can be absorbed by pushing it past $\mathcal{T}$ and letting it act on $P^{I \alpha}$. The Siegel transformations in the dual theory take the following form:

$$
\delta_{\kappa} \tilde{E}_{(\eta)}^{I \alpha}=\partial_{i} \tilde{Z}^{M} \tilde{E}_{(\eta)}{ }^{a}{ }^{a} P_{\mathcal{I}}{ }^{i j} \tilde{\kappa}_{(\eta) j}{ }^{I \beta}\left(\Gamma_{a} \Gamma_{-\eta}\right)_{\beta}{ }^{\alpha} \quad \mathcal{I}=\left\{\begin{array}{l}
+ \text { if } I=1 \\
- \text { if } I=2
\end{array}\right.
$$

From equation (3.35) we also see that both types of vielbein in the dual theory have the same chirality. Let us note that by redefining $\tilde{E}^{\alpha, \bar{\alpha}} \longleftrightarrow \tilde{E}^{1 \alpha} \mp i \tilde{E}^{2 \alpha}$ one recovers 3 the Siegel symmetry transformations of type IIB superstring theory as stated in [13].

All possible choices of bosonic vielbein can be summarized in the following diagram:

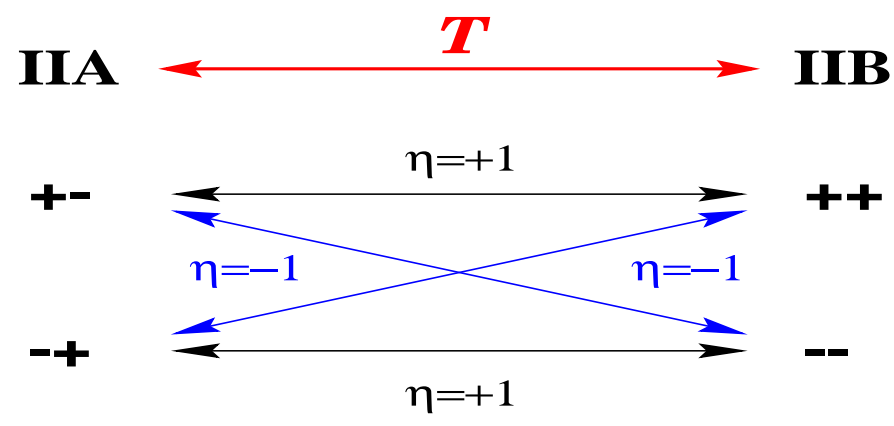

Fig. 1. T-dualities of type II string theories.

\footnotetext{
${ }^{3}$ In reference $[13$ the factor of $i$ in the above redefinition is absorbed in the spinor metric
} 
The + and - signs in the figure represent the chiralities of the two space-time supercharges, in the order $\left(Q^{1}, Q^{2}\right)$.

In order to fully determine the Buscher rules at the level of supervielbein we must now analyze the situation of $\tilde{E}_{(\eta)}{ }^{I \alpha}$. Since the Siegel transformations depend only on $\tilde{P}_{(\eta) \hat{M}}^{I \alpha}$ they cannot fix the functional form of $a_{(\eta)}^{I \alpha}(E)$. On the other hand $a_{(\eta)}^{I \alpha}$ must have the same chirality as $\tilde{P}_{(\eta) \hat{M}}^{I \alpha}$ since the latter contains $\tilde{E}_{(\eta)_{9}}{ }^{I \alpha}$ in its definition. Also, for $\tilde{\eta}=\eta$, the involution requirement implies the following:

$$
\tilde{E}_{(\tilde{\eta}) 9}^{I}{ }^{\alpha}=\tilde{a}_{(\tilde{\eta})}^{I \alpha}(E)=a_{(\eta)}^{I \alpha}\left(\tilde{E}_{\hat{M}}^{B}, \tilde{E}_{9}^{b}, \tilde{E}_{9}^{J \beta}\right)=a_{(\eta)}^{I \alpha}\left(\tilde{E}_{\hat{M}}^{A}, \tilde{E}_{9}{ }^{a}, a_{(\eta)}{ }^{J \beta}(E)\right)
$$

This equation has many independent solutions. At the same time, the superspace diffeomorphism covariance implies that $a^{\alpha}$ depends only on $E_{9}{ }^{A}$ since the parameters do not depend on the ninth bosonic coordinate and $\tilde{E}_{9}{ }^{A}$ should transform as the ninth component of a covariant vector. The index structure and chirality requirements for $\tilde{E}_{9}^{I \alpha}$ suggest that

$$
\tilde{E}_{(\eta) 9}{ }^{I \alpha}=\frac{E_{9}{ }^{J \beta}}{f_{(\eta) J}\left(G_{99}\right)} \mathcal{T}_{(\eta)_{J \beta}}{ }^{I \alpha} .
$$

From equation (3.38) we see that the function $f_{(\eta) J}$ has to be a monomial, but its exact expression is arbitrary. To completely fix the form of $f_{(\eta) J}$ we study in detail the supergravity constraints. We will require that the IIB supergravity constraints are satisfied if the IIA constraints are. As pointed out before, this is not an outside constraint, since the Siegel symmetry, having the right form, already produces the right conventional constraints as well as the other lower dimensional constraints, while the Bianchi identities are responsible for the rest.

\section{Matching the supergravity constraints}

In this section we will study the change in supergravity constraints under T-duality. We will first fix the arbitrariness in the functions $f_{(\eta) I}\left(G_{99}\right)$ by comparing the constraints computed with our dual fields (equations (3.5), (3.35) and (3.39)) with those given in [14. Then we compute the torsion constraints. As a nice consistency check we will notice that the dimension 0 constraints are satisfied if the IIA constraints are satisfied. From one of the consequences of Siegel symmetry we infer the dual spin connections. Then, the dimension 1/2 constraints will produce for us the duality transformation of the dilaton and its superpartner. The constraints of dimension 1 will generate the transformations of RR fields.

It turns out that the easiest way to fix $f_{(\eta) I}\left(G_{99}\right)$ is to study the dual NS-NS field strength. We will keep $\eta$ arbitrary for the time being. Later on we will fix it to $\eta=1$. To flatten the indices we need the inverse of the dual vielbein. It is given by:

$$
\tilde{E}_{a}^{\hat{M}}=E_{a}^{\hat{M}}-E_{9 a} E_{9}^{\alpha I} E_{\alpha I}^{\hat{M}} s_{(\eta) I}
$$




$$
\begin{aligned}
\tilde{E}_{a}{ }^{9} & =E_{9 a}\left(1+s_{(\eta) I} E_{9}{ }^{\alpha I} E_{\alpha I}{ }^{\hat{M}} B_{\hat{M} 9}\right)-E_{a}{ }^{\hat{M}} B_{\hat{M} 9} \\
\tilde{E}_{\alpha I}{ }^{\hat{M}} & =\mathcal{T}_{\alpha I}{ }^{\beta J} E_{\beta J}{ }^{\hat{M}} \quad ; \quad \tilde{E}_{\alpha I}{ }^{9}=-\mathcal{T}_{\alpha I}{ }^{\beta J} E_{\beta J}{ }^{\hat{M}} B_{\hat{M} 9}
\end{aligned}
$$

Here summation over the index $I$ is assumed and $s_{(\eta) I}=\frac{1}{f_{(\eta) I}\left(G_{99}\right)}-\frac{1}{G_{99}}$. Using this and equation (A.6) we get the following for the dual 3-form field strength:

$$
\tilde{F}_{A B C}=F_{A^{\prime} B^{\prime} C^{\prime}} \mathcal{T}_{A}^{A^{\prime}} \mathcal{T}_{B}^{B^{\prime}} \mathcal{T}_{C}^{C^{\prime}}-(A B C)-(-)^{A(B+C)}(B C A)-(-)^{C(A+B)}(C A B)
$$

with $(A B C)$ given by

$$
\begin{aligned}
(A B C) & =E_{9 a} \delta_{C}^{a}\left\{\frac{1}{G_{99}} E_{9}{ }^{d} F_{d A^{\prime} B^{\prime}} \mathcal{T}_{A}{ }^{A^{\prime}} \mathcal{T}_{B}{ }^{B^{\prime}}+\frac{1}{f_{(\eta) I}\left(G_{99}\right)} E_{9}{ }^{\alpha I} F_{\alpha I A^{\prime} B^{\prime}} \mathcal{T}_{A}{ }^{A^{\prime}} \mathcal{T}_{B}{ }^{B^{\prime}}\right. \\
& \left.+\frac{\eta}{G_{99}} \mathbb{T}_{A^{\prime} B^{\prime}}{ }^{a} E_{9 a} \mathcal{T}_{A}{ }^{A^{\prime}} \mathcal{T}_{B}{ }^{B^{\prime}}+\frac{\eta}{G_{99}} E_{9}{ }^{D} \mathbb{T}_{D B^{\prime} d} \delta_{A}^{d} \mathcal{T}_{B}{ }^{B^{\prime}}-\frac{\eta}{G_{99}} E_{9}{ }^{D} \mathbb{T}_{D A^{\prime} d} \delta_{B}^{d} \mathcal{T}_{A}{ }^{A^{\prime}}\right\}
\end{aligned}
$$

with $\mathbb{T}_{A B}{ }^{C}=T_{A B}{ }^{C}+E_{A}{ }^{N} \Omega_{N B}{ }^{C}-(-)^{A B} E_{B}{ }^{N} \Omega_{N A}{ }^{C}$.

The 3-form field strength constraints summarized in the Appendix set to zero the components $\tilde{F}_{I \alpha J \beta K \gamma}$ in both IIA and IIB theories. It is easy to check that this is trivially satisfied, since $E_{9 a} \delta_{A}^{a}=0$ if $A$ is not a bosonic index.

By considering the constraints on $\tilde{F}_{I \alpha J \beta c}$ we do not get any information about the form of $f_{(\eta) I}$, but we have a nontrivial consistency check for the results obtained from Siegel symmetry arguments.

In both IIA and IIB supergravity one requires that the field strength components with two bosonic and one fermionic indices vanishes. Imposing this on equations (4.2) and (4.3) amounts to canceling $\tilde{E}_{(\eta) 9}{ }^{K \rho} F_{K \rho I \gamma a}$ against $\frac{\eta}{G_{99}} E_{9}{ }^{K} \rho T_{K \rho I \gamma a}$. Using the IIA constraints we find that the functions $f_{(\eta) I}$ are given by

$$
f_{(\eta) 1}=-\eta G_{99} \quad f_{(\eta) 2}=\eta G_{99}
$$

which together with equation (3.36) recovers the results stated in equation (1.1-1.2). This and the Siegel symmetry imply that all the constraints of IIB supergravity are satisfied. We are therefore free to use them to derive the T-duality action on fields that do not appear explicitly in the sigma model action.

Now we turn to studying the torsion constraints. Since the formulas are cumbersome enough, we will set $\eta=1$. The $\eta=-1$ case can be recovered by interchanging the labels 1 and 2. We will also write $s_{I}$ for $s_{(1) I}$. For notational convenience we extend the index of $f$ to a full superspace index and define $f_{a}=G_{99}$. Likewise, the index of $s$ is extended to a full superspace index and $s_{a}=0$. First we write down the dual torsion in terms of the IIA variables. The dimension 0 components will provide additional consistency checks to our previous results, the dimension $1 / 2$ components will determine the dual spin $1 / 2$ fields as well the dimension $1 / 2$ dual spin connection, while the dimension 1 components will generate the rest of the dual spin connection components as well as the duality transformation of the RR fields. 
Using the definitions and the constraints listed in the appendix we find the following for the dual torsion:

$$
\begin{aligned}
& \tilde{\mathbb{T}}_{A B}{ }^{C}=\mathbb{T}_{A^{\prime} B^{\prime}}{ }^{C^{\prime}} \mathcal{T}_{A}{ }^{A^{\prime}} \mathcal{T}_{B}{ }^{B^{\prime}} \mathcal{T}_{C^{\prime}}{ }^{C}-\left\{\frac{1}{2 f_{C}} E_{9}{ }^{D} F_{D A^{\prime} B^{\prime}} E_{9}{ }^{C} \mathcal{T}_{A}{ }^{A^{\prime}} \mathcal{T}_{B}{ }^{B^{\prime}} \mathcal{T}_{C}{ }^{C^{\prime}}\right. \\
& -\frac{s_{I}}{f_{C}} E_{9 d} \delta_{A}^{d} E_{9}{ }^{I \alpha} E_{9}{ }^{D} F_{D I \alpha B^{\prime}} E_{9}{ }^{C^{\prime}} \mathcal{T}_{B}{ }^{B^{\prime}} \mathcal{T}_{C^{\prime}}{ }^{C} \\
& +\frac{1}{G_{99}} E_{9}{ }^{D} \mathbb{T}_{D B^{\prime} d} \delta_{A}^{d} E_{9}{ }^{C^{\prime}} \mathcal{T}_{B}{ }^{B^{\prime}} \mathcal{T}_{C^{\prime}}{ }^{C} \\
& +\frac{1}{2 G_{99}} \mathbb{T}_{A^{\prime} B^{\prime}}{ }^{a} E_{9 a} E_{9}{ }^{C^{\prime}} \mathcal{T}_{A}{ }^{A^{\prime}} \mathcal{T}_{B}{ }^{B^{\prime}} \mathcal{T}_{C^{\prime}}{ }^{C} \\
& +s_{C} E_{9}{ }^{D} \mathbb{T}_{D A^{\prime}}{ }^{C^{\prime}}\left(E_{9}\right)_{d} \delta_{B}^{d} \mathcal{T}_{A}{ }^{A^{\prime}} \mathcal{T}_{C^{\prime}}{ }^{C} \\
& -\frac{s_{I}}{G_{99}} E_{9 d} \delta_{A}^{d} E_{9}{ }^{I \alpha} \mathbb{T}_{I \alpha B^{\prime}}{ }^{b} E_{9 b} E_{9}{ }^{C^{\prime}} \mathcal{T}_{B}{ }^{B^{\prime}} \mathcal{T}_{C^{\prime}}{ }^{C} \\
& -\frac{s_{I}}{G_{99}} E_{9}{ }^{I \alpha} E_{9}{ }^{D} \mathbb{T}_{D I \alpha d} \delta_{A}^{d} E_{9 d} \delta_{B}^{d} E_{9}{ }^{C^{\prime}} \mathcal{T}_{C^{\prime}}{ }^{C} \\
& +\partial_{A^{\prime}}\left(\frac{1}{f_{C}} \mathcal{T}_{C^{\prime}}{ }^{C}-\frac{1}{G_{99}}\right) E_{9 d} \delta_{B}^{d} E_{9}{ }^{\prime} \mathcal{T}_{A}{ }^{\prime} \\
& +s_{I} E_{9 d} \delta_{A}^{d} E_{9}^{I \alpha} \mathbb{T}_{I \alpha B^{\prime}}{ }^{\prime \prime} \mathcal{T}_{B}{ }^{B^{\prime}} \mathcal{T}_{C^{\prime}}{ }^{C} \\
& +\frac{1}{\sqrt{G_{99}}} E_{9}{ }^{D} \mathbb{T}_{D A^{\prime}}{ }^{a} \delta_{B^{\prime}}{ }^{2 \alpha}\left(\Gamma_{a} \Gamma_{+}\right)_{\alpha}{ }^{\beta} \delta_{2 \beta}{ }^{C} \mathcal{T}_{A}{ }^{A^{\prime}} \mathcal{T}_{B}{ }^{B^{\prime}} \\
& -\frac{1}{G_{99}} E_{9}{ }^{D} \mathbb{T}_{D A^{\prime}}{ }^{a}\left(E_{9}\right)_{a} \delta_{B}{ }^{2 \alpha} \delta_{2 \alpha}{ }^{C} \mathcal{T}_{A}{ }^{A^{\prime}} \\
& -\frac{1}{\sqrt{G_{99}}} \frac{1}{f_{2 \alpha}} E_{9}{ }^{D} \mathbb{T}_{D A^{\prime}}{ }^{a} E_{9}{ }^{2 \alpha}\left(\Gamma_{a} \Gamma_{+}\right)_{\alpha}{ }^{\beta} \delta_{2 \beta}{ }^{C} E_{9 d} \delta_{B}^{d} \mathcal{T}_{A}{ }^{\prime} \\
& +\frac{1}{G_{99}} \frac{1}{f_{2 \alpha}} E_{9}{ }^{D} \mathbb{T}_{D A^{\prime}}{ }^{a} E_{9 a} E_{9 d} \delta_{B}^{d} E_{9}{ }^{2 \alpha} E_{\alpha}{ }^{\beta} \delta_{2 \beta}{ }^{C} \mathcal{T}_{A} A^{\prime} \\
& -\frac{s_{I}}{\sqrt{G_{99}}} E_{9}{ }^{\gamma I} E_{9}{ }^{D} \mathbb{T}_{D \gamma I}{ }^{a}{\delta_{B^{\prime}}}^{2 \alpha}\left(\Gamma_{a} \Gamma_{+}\right)_{\alpha}{ }^{\beta} \delta_{2 \beta}{ }^{C} E_{9 d} \delta_{A}^{d} \mathcal{T}_{B}{ }^{B^{\prime}} \\
& \left.+\frac{s_{I}}{G_{99}} E_{9}{ }^{\gamma I} E_{9}{ }^{D} \mathbb{T}_{D \gamma I}{ }^{a} E_{9 a} E_{9 d} \delta_{A}^{d} \delta_{B}{ }^{2 \alpha} \delta_{2 \alpha}{ }^{C}\right\}+(-)^{A B}(B A)
\end{aligned}
$$

As usual, the spin connection with fermionic indices (which appear in $\tilde{\mathbb{T}}$ and $\mathbb{T}$ ) is related to the one with bosonic Lorentz indices by

$$
\Omega_{A \alpha}^{\beta}=\frac{1}{8} \Omega_{A, a}^{b}\left(\sigma_{b}^{a}\right)_{\alpha}^{\beta}
$$

where, as stated in the Appendix, $\sigma^{a b}$ contains no factor of $1 / 2$.

As promised earlier, equation (4.5) will provide the T-duality transformations of the remaining (super)fields as well as more consistency checks. Starting with torsion components of dimension 0 and writing the result in 16-component notation, we find that

$$
\tilde{T}_{1 \alpha 2 \beta}{ }^{a}=0 \quad ; \quad \tilde{T}_{1 \alpha 1 \beta}{ }^{a}=i\left(\sigma^{a}\right)_{\alpha \beta} \quad ; \quad \tilde{T}_{2 \alpha 2 \beta}{ }^{a}=i\left(\sigma^{a}\right)_{\alpha \beta} .
$$

These are indeed the correct constraints of IIB supergravity as shown in [14] and summarized in the Appendix. 
We proceed by finding the relation between the spin connections in the type II theories. They arise from constraints of dimension $1 / 2$ and 1 . The fact that the Siegel symmetry has the correct form implies that $\tilde{T}_{a b}{ }^{c}=0$ and $\tilde{T}_{I \alpha b}{ }^{c}=0$. Using this information in equation (4.5) we find that

$$
\tilde{\Omega}_{1 \alpha b}^{a}=\Omega_{1 \alpha b}{ }^{a}+i s_{1} E_{9}{ }^{1 \beta}\left(\left(\Gamma^{a} \Gamma_{+} C^{-1}\right)_{\beta \alpha} E_{9 b}-\left(\Gamma_{b} \Gamma_{+} C^{-1}\right)_{\beta \alpha} E_{9}{ }^{a}\right) \quad \tilde{\Omega}_{2 \alpha b}^{a}=\not_{\alpha}{ }^{\beta} \Omega_{2 \beta b}{ }^{a}
$$

and

$$
\begin{aligned}
\tilde{\Omega}_{[a b]}{ }^{c}= & \Omega_{[a b]}{ }^{c}-\frac{1}{G_{99}} E_{9}{ }^{d} \Omega_{[d b] a} E_{9}{ }^{c}+\frac{1}{G_{99}} E_{9}{ }^{d} \Omega_{[d a] b} E_{9}{ }^{c}-\frac{1}{G_{99}} \Omega_{[a b] d} E_{9}{ }^{d} E_{9}{ }^{c} \\
& -\frac{1}{G_{99}} E_{9}{ }^{d} F_{d a b} E_{9}{ }^{c}-\frac{2}{G_{99}} E_{9}{ }^{I \alpha} \Omega_{I \alpha b a} E_{9}{ }^{c}+\frac{2}{G_{99}} E_{9}{ }^{1 \alpha} E_{9[a \mid} \Omega_{1 \alpha \mid b]}{ }^{c} .
\end{aligned}
$$

From the last equation follows $\Omega_{a b}{ }^{c}$ as

$$
\Omega_{a b}^{c}=\frac{1}{2}\left(\Omega_{[a b]}^{c}+\Omega_{[c a]}^{b}-\Omega_{[b c]}^{a}\right) .
$$

Knowing the spin connections it is now easy to proceed and find the dual spin $1 / 2$ fields. They come from constraints of dimension $1 / 2$. From $\tilde{T}_{1 \alpha 1 \beta}{ }^{\gamma 1}$ and $\tilde{T}_{2 \alpha 2 \beta}{ }^{\gamma 2}$ follows that $\tilde{\Lambda}_{I \alpha}$ are given by:

$$
\begin{aligned}
& 2 \tilde{\Lambda}_{1 \alpha}=\quad \Lambda_{1 \alpha}-\partial_{1 \alpha} \ln \left(G_{99}\right)^{-1 / 2}=\Lambda_{1 \alpha}+i\left(G_{99}\right)^{-1 / 2} E_{9}{ }^{\gamma 1}\left(\not C C^{-1}\right)_{\gamma \alpha} \\
& 2 \tilde{\Lambda}_{2 \alpha}=E_{\alpha}{ }_{\alpha}^{\dot{\beta}}\left(\Lambda_{2 \beta}-\partial_{2 \beta} \ln \left(G_{99}\right)^{-1 / 2}\right)=\left(\not H \Lambda_{2}\right)_{\alpha}-i\left(G_{99}\right)^{-1 / 2} E_{9}{ }^{\gamma 2}\left(C^{-1}\right)_{\alpha \dot{\gamma}}
\end{aligned}
$$

Since $\tilde{\Lambda}_{\alpha}=\tilde{E}_{\alpha}{ }^{M} \partial_{M} \tilde{\phi}$ it follows that the dual dilaton is given by

$$
2 \tilde{\phi}=\phi-\ln \left(G_{99}\right)^{-1 / 2}
$$

This leads us to interpret $2 \tilde{\phi}$ of reference [14 as the type IIB dilaton and thus get the standard dilaton shift under T-duality, as shown in [1]. Opposite to that paper and as noted in [10], in this treatment the dilaton shift appears at the classical level. This can be intuitively understood from the fact that supergravity is already a one loop effect from the sigma model point of view.

The constraints of dimension 1 , besides determining the transformation rules of RR fields, provide some more consistency checks. In particular, it can be shown that, upon using (4.5) together with

$$
\begin{aligned}
\tilde{F}_{a b c} & =\frac{1}{G_{99}}\left[-E_{9}{ }^{d} F_{d a b}\left(E_{9}\right)_{c}-\Omega_{a b}{ }^{d}\left(E_{9}\right)_{d}\left(E_{9}\right)_{c}\right. \\
& \left.-\left(E_{9}\right)^{D} \Omega_{D b a}\left(E_{9}\right)_{c}+\left(E_{9}\right)^{D} \Omega_{D a b}\left(E_{9}\right)_{c}\right]_{[a b c]}
\end{aligned}
$$

and equation (4.11), imply that the following equations are satisfied:

$$
\tilde{T}_{a 1 \alpha}{ }^{1 \beta}=-\frac{1}{16}\left(\Gamma^{b c} \Gamma_{-}\right)_{\alpha}{ }^{\beta} \tilde{F}_{a b c} \quad \tilde{T}_{a 2 \alpha}{ }^{2 \beta}=\frac{1}{16}\left(\Gamma^{b c} \Gamma_{-}\right)_{\alpha}{ }^{\beta} \tilde{F}_{a b c} .
$$


These are nothing but equations (A.22) from the list of IIB constraints in the Appendix.

The RR (super)field transformations follow from the $\tilde{T}_{a 1 \alpha}{ }^{2 \beta}$ and $\tilde{T}_{a 2 \alpha}{ }^{1 \beta}$ components of equation (4.5). They are given by:

-the axion

$$
\begin{aligned}
-i \nabla_{b}(W-\bar{W}) & =\frac{1}{2} E_{9}{ }^{c} F_{c b}-\frac{1}{4} e^{\phi} E_{9}{ }^{d} \Lambda_{1 \alpha}\left(C \Gamma_{d b} \Gamma_{+}\right)^{\alpha \beta} \Lambda_{2 \beta}+\frac{7}{4} e^{2 \tilde{\phi}} \tilde{\Lambda}_{1 \alpha}\left(C \Gamma_{b} \Gamma_{-}\right)^{\alpha \beta} \tilde{\Lambda}_{2 \beta} \\
+ & e^{\phi} \frac{E_{9 b}}{2 G_{99}} E_{9}{ }^{1 \alpha}\left(\Gamma_{-} C^{-1}\right)_{\alpha \beta} E_{9}{ }^{2 \beta}-e^{\phi} \frac{E_{9}{ }^{d}}{4 G_{99}} E_{9}{ }^{1 \alpha}\left(\Gamma_{-} \Gamma_{d b} C^{-1}\right)_{\alpha \beta} E_{9}{ }^{2 \beta}
\end{aligned}
$$

-the RR 3-form field strength

$$
\begin{aligned}
-i\left(G_{b c d}-\bar{G}_{b c d}\right)-i(W-\bar{W}) \tilde{F}_{b c d} & =\frac{1}{4} E_{9[b} F_{c d]}-E_{9}{ }^{a} F_{a b c d}^{\prime} \\
& -\frac{e^{\phi}}{8 G_{99}} E_{9[b} E_{9}^{1 \alpha}\left(\Gamma_{-} \Gamma_{c d]} C^{-1}\right)_{\alpha \beta} E_{9}{ }^{2 \beta} \\
& +\frac{e^{\phi}}{24 ! G_{99}} E_{9}{ }^{a} E_{9}{ }^{1 \alpha}\left(\Gamma_{-} \Gamma_{a b c d} C^{-1}\right)_{\alpha \beta} E_{9}{ }^{2 \beta} \\
& -\frac{1}{8} e^{\phi} E_{9[b} \Lambda_{1 \alpha}\left(C \Gamma_{c d]} \Gamma_{+}\right)^{\alpha \beta} \Lambda_{2 \beta} \\
& -\frac{1}{24 !} e^{\phi} E_{9}{ }^{a} \Lambda_{1 \alpha}\left(C \Gamma_{a b c d} \Gamma_{+}\right)^{\alpha \beta} \Lambda_{2 \beta} \\
& +\frac{1}{3} e^{2 \tilde{\phi}} \tilde{\Lambda}_{1 \alpha}\left(C \Gamma_{b c d} \Gamma_{-}\right)^{\alpha \beta} \tilde{\Lambda}_{2 \beta}
\end{aligned}
$$

which together with equation (4.14) fixes the imaginary part of $G_{a b c}$, i.e. the RR 3-form field strength.

-the RR 5-form field strength

$$
\begin{aligned}
& \tilde{F}_{a b c d e}=\frac{-1}{24 !}\left[E_{9[a} F_{b c d e]}^{\prime}+\frac{1}{5 !} \epsilon_{a b c d e}{ }^{f g h i j} E_{9[f} F^{\prime}{ }_{g h i j]}\right]-\frac{e^{2 \tilde{\phi}}}{85 !} \tilde{\Lambda}_{1 \alpha}\left(C \Gamma_{a b c d e} \Gamma_{-}\right)^{\alpha \beta} \tilde{\Lambda}_{2 \beta} \\
& +\frac{e^{\phi}}{4(4 !)^{2} G_{99}}\left[E_{9[a} E_{9}^{1 \alpha}\left(\Gamma_{-} \Gamma_{b c d e]} C^{-1}\right)_{\alpha \beta} E_{9}{ }^{2 \beta}+\frac{1}{5 !} \epsilon_{a b c d e}{ }^{i j k l m} E_{9[i} E_{9}{ }^{1 \alpha}\left(\Gamma_{-} \Gamma_{j k l m]} C^{-1}\right)_{\alpha \beta} E_{9}{ }^{2 \beta}\right] \\
& +\frac{e^{\phi}}{4(4 !)^{2}}\left[E_{9[a} \Lambda_{1 \alpha}\left(C \Gamma_{b c d e]} \Gamma_{+}\right)^{\alpha \beta} \Lambda_{2 \beta}+\frac{1}{5 !} \epsilon_{a b c d e}{ }^{i j k l m} E_{9[i} \Lambda_{1 \alpha}\left(C \Gamma_{j k l m]} \Gamma_{+}\right)^{\alpha \beta} \Lambda_{2 \beta}\right]
\end{aligned}
$$

This equation provides another consistency check. By simple inspection one can easily check that $\tilde{F}_{\text {abcde }}$ is self-dual, as it should be.

\section{Conclusions}

This completes the construction of the T-duality action on the massless superfields of type II superstring theory, to all orders in the odd superspace coordinates. The results 
are summarized in equations (1.1-1.2) for the supervielbein and super 2-form, in equation (4.12) for spin 1/2 fermions and in equations (4.16), 4.17) and (4.19) for the RR superfields.

It is easy to see that the dual gravitini obtained here agree with those derived in [8]. We have also constructed the T-duality transformations of the RR field strengths by studying the constraints of the type II supergravity theories. Another way of rederiving them would be to expand the supervielbein and super 2-form in the odd superspace coordinates. The in principle, results should agree with those obtained in [10], since the duality transformation commutes with the series expansion. However, possible discrepancies can be associated to differences in the space-time interpretation of the fields.

The approach taken in this paper has the advantage of producing the action of Tduality on all the space-time fermions without explicit reference to space-time supersymmetry transformation rules and, at the same time, providing a compact form of all dual space-time fields.

An interesting exercise would be to relax the constraint that all fields in the original theory are independent of $X^{9}$ by replacing it with isometries characterized by some Killing vectors $k_{M}$. Such an approach should confirm once again the $S O(d, d ; \mathbb{Z})$ [17] structure of T-duality for IIA/B theory on a manifold with $d$ Killing vectors and should allow the construction of these transformations to all orders in the odd superspace variables.

\section{Acknowledgment}

We would like to thank Martin Roček for suggesting this problem and for usefull suggestions and discussions. This work was supported in part by the NSF grant PHY-9722101.

\section{Appendix}

In this appendix we list the conventions we are using as well as the IIA and (appropriately redefined) IIB supergravity constraints.

We use both 16 and 32-component notation. $\Gamma$ matrices are defined in terms of two sets of generalized Pauli matrices $\left(\sigma_{a}\right)_{\alpha}{ }^{\dot{\beta}}$ and $\left(\bar{\sigma}_{a}\right)_{\dot{\alpha}}{ }^{\beta}$ that have the following anticommutation relations:

$$
\sigma_{(a}, \bar{\sigma}_{b)}=-2 \eta_{a b}
$$

with the "mostly plus" metric. The 16-dimensional indices are raised and lowered with the charge conjugation matrix $\mathcal{C}^{\alpha \dot{\beta}}$ and its inverse $\mathcal{C}_{\alpha \dot{\beta}}$ :

$$
\left(\sigma_{a}\right)_{\alpha \beta}=\left(\sigma_{a}\right)_{\alpha}{ }^{\dot{\beta}} \mathcal{C}_{\beta \dot{\beta}} \quad\left(\bar{\sigma}_{a}\right)_{\dot{\alpha} \dot{\beta}}=\left(\bar{\sigma}_{a}\right)_{\dot{\alpha}}{ }^{\beta} \mathcal{C}_{\beta \dot{\beta}} \quad \mathcal{C}^{\alpha \dot{\beta}} \mathcal{C}_{\alpha \dot{\gamma}}=\delta_{\dot{\gamma}}^{\dot{\beta}}
$$

The antisymmetric product of $n \sigma$ matrices, $\sigma^{(n)}$ used here, and in general the antisymmetric product of $n$ objects, without a factor of $\frac{1}{n !}$, e.g.

$$
\left(\sigma_{a b}\right)_{\alpha}{ }^{\beta}=-\left(\sigma_{a}\right)_{\alpha}{ }^{\dot{\beta}}\left(\bar{\sigma}_{b}\right)_{\dot{\beta}}{ }^{\beta}+\left(\sigma_{b}\right)_{\alpha}{ }^{\dot{\beta}}\left(\bar{\sigma}_{a}\right)_{\dot{\beta}}{ }^{\beta}
$$


$\left(\sigma_{a}\right)_{\alpha \beta},\left(\sigma_{(5)}\right)_{\alpha \beta}$ are symmetric in spinor indices while $\left(\sigma_{(3)}\right)_{\alpha \beta}$ is antisymmetric. The barred version of the matrices have the same symmetry properties as the unbarred ones and carry dotted indices. We will occasionally drop the bars in situations where there is no possibility of confusion.

$\Gamma$ matrices are constructed as

$$
\Gamma_{\alpha}^{\beta}=\left(\begin{array}{cc}
0 & i \bar{\sigma}_{\dot{\alpha}}^{\beta} \\
i \sigma_{\alpha}{ }^{\dot{\beta}} & 0
\end{array}\right)
$$

and satisfy the usual anticommutation relations $\left\{\Gamma^{a}, \Gamma^{b}\right\}=2 \eta^{a b}$.

In 32-component notation one can choose from two possible charge conjugation matrices, $C_{+}$and $C_{-}$, which differ by a factor of $\Gamma_{11}$. We choose to work with $C \equiv C_{+}$, and the associated identities are:

$$
C^{T}=-C \quad \Gamma_{a}^{T}=-C \Gamma_{a} C^{-1} \quad C^{\dagger}=C^{-1}
$$

One can go back and forth between 16 and 32-component notations by inserting the appropriate chiral projectors as well as charge conjugation matrices.

The general expression for torsion and 2-form field strength are :

$$
\begin{aligned}
T_{A B}{ }^{C}= & (-)^{B N} E_{A}{ }^{N} E_{B}{ }^{M}\left(\partial_{M} E_{N}{ }^{C}-(-)^{M N} \partial_{N} E_{M}{ }^{C}\right)-E_{A}{ }^{N} \Omega_{N B}{ }^{C}+(-)^{A B} E_{B}{ }^{N} \Omega_{N A}{ }^{C} \\
F_{A B C}= & -(-)^{C(M+N)+B M} E_{A}{ }^{M} E_{B}{ }^{N} E_{C}{ }^{K}\left(\partial_{K} B_{M N}\right. \\
& \left.\quad+(-)^{K(M+N)} \partial_{M} B_{N K}+(-)^{N(K+M)} \partial_{N} B_{K M}\right) \quad(\text { A.6) }
\end{aligned}
$$

With these conventions, the IIA constraints are [14]:

$$
\begin{aligned}
& T_{1 \alpha 1 \beta}^{a}=i\left(\sigma^{a}\right)_{\alpha \beta} \quad T_{2 \alpha 2 \beta}{ }^{a}=i\left(\sigma^{a}\right)_{\dot{\alpha} \dot{\beta}} \quad T_{1 \alpha 2 \beta}{ }^{a}=0 \\
& F_{a 1 \alpha 1 \beta}=-i\left(\sigma_{a}\right)_{\alpha \beta} \quad F_{a 2 \alpha 2 \beta}=i\left(\sigma^{a}\right)_{\dot{\alpha} \dot{\beta}} \quad F_{a 1 \alpha 2 \beta}=0 \\
& F_{a b I \gamma}=0 \quad T_{I \alpha b}{ }^{c}=0 \\
& T_{1 \alpha 1 \beta}^{\gamma 1}=\left[\delta_{\left(\alpha^{\gamma} \delta_{\beta)}\right.}^{\delta}+\left(\sigma^{a}\right)_{\alpha \beta}\left(\sigma_{a}\right)^{\gamma \delta}\right] \Lambda_{\delta 1} \\
& T_{2 \alpha 2 \beta}{ }^{\gamma 2}=\left[\delta_{(\dot{\alpha}} \dot{\gamma} \delta_{\dot{\beta})}^{\dot{\delta}}+\left(\sigma^{a}\right)_{\dot{\alpha} \dot{\beta}}\left(\sigma_{a}\right)^{\dot{\gamma} \dot{\delta}}\right] \Lambda_{\delta 2} \\
& T_{1 \alpha 1 \beta}{ }^{\gamma 2}=T_{1 \alpha 2 \beta}^{\gamma 1}=T_{1 \alpha 2 \beta}{ }^{\gamma 2}=T_{2 \alpha 2 \beta^{\gamma 1}}=0 \\
& T_{a 1 \beta}^{1 \alpha}=-\frac{1}{16}\left(\sigma_{b c}\right)_{\beta}{ }^{\alpha} F_{a b c} \quad T_{a 2 \beta}{ }^{2 \alpha}=\frac{1}{16}\left(\sigma_{b c}\right)_{\dot{\beta}}^{\dot{\alpha}} F_{a b c} \\
& T_{a 1 \alpha}{ }^{2 \beta}=-\frac{i}{16}\left(\sigma_{a}\right)_{\alpha \gamma}\left[\frac{1}{2}\left(\sigma^{b c}\right)^{\dot{\beta} \gamma} B_{b c}-\frac{1}{12} \frac{1}{4 !}\left(\sigma^{b c d e}\right)^{\dot{\beta} \gamma} D_{b c d e}\right] \\
& T_{a 2 \alpha}^{1 \beta}=\frac{i}{16}\left(\sigma_{a}\right)_{\dot{\alpha} \dot{\gamma}}\left[\frac{1}{2}\left(\sigma^{b c}\right)^{\dot{\gamma} \beta} B_{b c}-\frac{1}{12} \frac{1}{4 !}\left(\sigma^{b c d e}\right)^{\dot{\gamma} \beta} D_{b c d e}\right] \\
& B_{b c}=e^{-\phi} F_{b c}-\frac{1}{2} \Lambda_{1 \beta}\left(\sigma_{b c}\right)^{\dot{\alpha} \beta} \Lambda_{2 \alpha}
\end{aligned}
$$




$$
D_{b c d e}=2 e^{-\phi} F_{b c d e}^{\prime}+\frac{1}{4 !} \Lambda_{1 \beta}\left(\sigma_{b c d e}\right)^{\dot{\alpha} \beta} \Lambda_{2 \alpha}
$$

The IIB constraints are also listed in [14], together with the appropriate field redefinitions which transform them to string frame. Redefining the upper indices as $1 \alpha=\frac{\alpha+\bar{\alpha}}{\sqrt{2}}$, $2 \alpha=-i \frac{\alpha-\bar{\alpha}}{\sqrt{2}}$ and the lower indices as $1 \alpha=\frac{\alpha+\bar{\alpha}}{\sqrt{2}}, 2 \alpha=i \frac{\alpha-\bar{\alpha}}{\sqrt{2}}$ they are $\frac{\alpha}{\text { : }}$ :

$$
\begin{aligned}
& T_{1 \alpha 1 \beta}{ }^{a}=i\left(\sigma^{a}\right)_{\alpha \beta} \quad T_{2 \alpha 2 \beta}{ }^{a}=i\left(\sigma^{a}\right)_{\alpha \beta} \quad T_{1 \alpha 2 \beta}{ }^{a}=0 \\
& F_{a 1 \alpha 1 \beta}=-i\left(\sigma_{a}\right)_{\alpha \beta} \quad F_{a 2 \alpha 2 \beta}=i\left(\sigma^{a}\right)_{\alpha \beta} \quad F_{a 1 \alpha 2 \beta}=0 \\
& F_{a b I \gamma}=0 \quad T_{I \alpha b}{ }^{c}=0 \\
& T_{I \alpha \beta J^{\gamma K}}^{\gamma K}=2\left[\delta_{(\alpha}^{\gamma} \delta_{\beta)}^{\delta}+\left(\sigma^{a}\right)_{\alpha \beta}\left(\sigma_{a}\right)^{\gamma \delta}\right] \Lambda_{\delta I} \delta_{I, J} \delta_{I, K} \\
& T_{a 1 \beta}^{1 \alpha}=-\frac{1}{16}\left(\sigma_{b c}\right)_{\beta}{ }^{\alpha} F_{a b c} \quad T_{a 2 \beta}{ }^{2 \alpha}=\frac{1}{16}\left(\sigma_{b c}\right)_{\beta}{ }^{\alpha} F_{a b c} \\
& T_{a 2 \alpha}^{1 \beta}=-\frac{i}{4}\left(\sigma_{a} \sigma^{b}\right)_{\alpha}{ }^{\beta}\left[e^{-2 \phi} \nabla_{b}(W-\bar{W})+\frac{7}{4} \Lambda_{\gamma 2}\left(\sigma_{b}\right)^{\gamma \delta} \Lambda_{\delta 1}\right] \\
& \text { - } \frac{1}{4 ! 48}\left(\sigma_{b c d e}\right)_{\alpha}{ }^{\beta}\left[\frac{5}{3} e^{-2 \phi} F_{a}{ }^{b c d e}+\frac{i}{5 ! 8} \Lambda_{\gamma 2}\left(\sigma_{a}^{b c d e}\right)^{\gamma \delta} \Lambda_{\delta 1}\right] \\
& +\frac{i}{4 ! 6}\left(\sigma_{a} \sigma^{b c d}\right)_{\alpha}{ }^{\beta}\left[e^{-2 \phi}\left(\bar{G}_{b c d}-G_{b c d}-(W-\bar{W}) F_{b c d}\right)+\frac{1}{3} \Lambda_{\gamma 2}\left(\sigma_{b c d}\right)^{\gamma \delta} \Lambda_{\delta 1}\right] \\
& T_{a 1 \alpha}{ }^{2 \beta}=\frac{i}{4}\left(\sigma_{a} \sigma^{b}\right)_{\alpha}{ }^{\beta}\left[e^{-2 \phi} \nabla_{b}(W-\bar{W})+\frac{7}{4} \Lambda_{\gamma 2}\left(\sigma_{b}\right)^{\gamma \delta} \Lambda_{\delta 1}\right] \\
& +\frac{1}{4 ! 48}\left(\sigma_{b c d e}\right)_{\alpha}{ }^{\beta}\left[\frac{5}{3} e^{-2 \phi} F_{a}^{b c d e}+\frac{i}{5 ! 8} \Lambda_{\gamma 2}\left(\sigma_{a}^{b c d e}\right)^{\gamma \delta} \Lambda_{\delta 1}\right] \\
& +\frac{i}{4 ! 6}\left(\sigma_{a} \sigma^{b c d}\right)_{\alpha}{ }^{\beta}\left[e^{-2 \phi}\left(\bar{G}_{b c d}-G_{b c d}-(W-\bar{W}) F_{b c d}\right)+\frac{1}{3} \Lambda_{\gamma 2}\left(\sigma_{b c d}\right)^{\gamma \delta} \Lambda_{\delta 1}\right]
\end{aligned}
$$

For both IIA and IIB theories, higher dimensional constraints can be constructed by using the lower dimensional ones together with the Bianchi identities [14], [18].

\section{References}

[1] T. Buscher, Phys.Lett. B159 (1985) 127; B194 (1987) 59; B201 (1988) 466

[2] K. Kikkawa, M. Yamasaki Phys.Lett. B149 (1984) 357

N. Sakai, I. Senda Prog.Theor.Phys.75 (1986) 692, Erratum-ibid.77, (1987) 773

[3] E. Bergshoeff, C.M. Hull, T. Ortin, Nucl.Phys. B451 (1995) 547

\footnotetext{
${ }^{4}$ The index redefinition is necessary for the Siegel transformations to have the form derived in section $\mathbf{3}$. The upper and lower indices are redefined in a different way for the scalar product to be preserved.
} 
[4] E. Bergshoeff, I. Entrop, R. Kallosh, Phys.Rev. D49 (1994) 6663

[5] M. Abou-Zeid, B. de Wit, D. Lüst, H. Nicolai, hep-th/9908169,

Phys.Lett. B466 (1999) 144

[6] J. Polchinski, hep-th/9611050

[7] J. H. Schwarz, Phys. Lett. B360 (1995) 13, hep-th/9508143; Erratum: ibid, B364 (1995) 252; Nucl. Phys. Proc. Suppl. 49 (1996) 183, hep-th/9509148; Phys. Lett. B367 (1996) 97, hep-th/9510086;

P. S. Aspinwall, Nucl. Phys. Proc. Suppl. 46 (1996) 30, hep-th/9508154].

[8] S.F. Hassan, Nucl.Phys. B460 (1996) 362

[9] S.F. Hassan, hep-th/9907152, Nucl.Phys. B568 (2000) 145

[10] M. Cvetič, H. Lü, C.N. Pope, K.S. Stelle, hep-th/9907202,

Nucl.Phys. B573 (2000) 149

[11] W. Siegel, Phys.Rev. D48 (1993) 2826 ; Phys.Rev. D47 (1993) 5453

[12] E. Bergshoeff, E. Sezgin, P.K. Townsend, Phys.Lett. B189 (1987) 75

[13] M.T. Grisaru, P. Howe, L. Mezincescu, B.E.W. Nilsson, P.K. Townsend, Phys.Lett. B162 (1985) 116

[14] S. Bellucci, S.James Gates, Jr., B. Radak, Sh. Vashakidze Mod.Phys.Lett. A4 (1989) 1985

[15] M.J. Duff, P.S. Howe, T. Inami, K.S. Stelle, Phys.Lett. 191B (1987) 70

[16] M. Roček, E. Verlinde, Nucl.Phys. B373 (1992) 630 and references therein

[17] A. Giveon, M. Rocek, Nucl.Phys. B380 (1992) 128

[18] P.S. Howe, P.C. West, Nucl.Phys. B238, (1984), 181 